\title{
Estrategia didáctica para aprender modelaje probabilístico en sistemas de colas
}

\section{Didactic strategy to learn probabilistic modeling in queuing sytems}

\author{
Víctor Miguel Burbano Pantoja ${ }^{1}$ \\ Margoth Adriana Valdivieso Miranda ${ }^{2}$ \\ Ángela Saray Burbano Valdivieso ${ }^{3}$ \\ UPTC
}

\section{RESUMEN}

Este trabajo investigativo tuvo como objetivo implementar una estrategia didáctica para elaborar un modelaje del sistema de colas del kiosco estudiantil en una universidad colombiana mediante modelos probabilísticos.

$1 \quad$ Magíster en Estadística, Doctor en Ciencias de la Educación. Escuela de Matemáticas y Estadística. Universidad Pedagógica y Tecnológica de Colombia, Tunja, Colombia. Docente investigador Grupo de investigación Interdisciplinario en Ciencias GICl. E-mail: victor.burbano@uptc.edu. co ORCID: https://orcid.org/0000-0002-3561-1886

2 Licenciada en matemáticas, Magíster en Ciencias-Estadística. Filiación: Universidad Pedagógica y Tecnológica de Colombia, Tunja, Colombia. Docente investigador Grupo de investigación Interdisciplinario en Ciencias GICl. E-mail: margoth.valdivieso@uptc.edu.co ORCID: https://orcid. org/0000-0002-3617-928X

3 Tecnóloga en programación de sistemas informáticos. Bióloga, Universidad Pedagógica y Tecnológica de Colombia (UPTC), Tunja, Colombia. Investigadora Grupo de investigación Interdisciplinario en Ciencias GICl. E-mail angela. burbano@uptc.edu.co

${ }^{*}$ Este trabajo se desprende de un proyecto de investigación avalado por la VIE de la UPTC.
La metodología fue cuantitativa con diseño de corte transversal. Los datos fueron recopilados por observación directa y conteo del número de usuarios que llegaban a la cola del kiosco universitario para adquirir sus tiquetes de almuerzo, en intervalos de 10 minutos. Los resultados evidenciaron que tal sistema podía modelarse mediante distribuciones de probabilidad de Poisson y exponencial. Este operaba de forma estable, el tiempo de espera en la cola era reducido y el tiempo de servicio fue pertinente. Se concluye que el modelamiento matemático es una herramienta poderosa para representar un sistema real pero requiere desarrollar una estrategia planificada que va de los datos hacia el modelo y de este nuevamente a la realidad. 


\section{PALABRAS CLAVE:}

sistemas de colas, modelaje matemático, distribuciones de probabilidad

\section{ABSTRACT}

This investigative work aimed to implement a didactic strategy to develop a modeling of the queue system of the student kiosk in a Colombian university using probabilistic models. The methodology was quantitative with a crosssectional design. The data was collected by direct observation and counting the number of users who reached the queue of the university kiosk to purchase their lunch tickets, in 10-minute intervals. The results showed that such a system could be modeled by Poisson and exponential probability distributions, this system operated in a stable way, the waiting time in the queue was reduced and the service time was relevant. It is concluded that mathematical modeling is a powerful tool to represent a real system but it requires developing a planned strategy that goes from the data to the model and from this back to reality.

\section{KEYWORDS:}

queuing systems, mathematical modeling, probability distributions

\section{INTRODUCCIÓN}

Con frecuencia en la enseñanza de la matemática se usan modelos abstractos que en su forma analítica incluyen una variedad de símbolos; los estudios sobre modelación matemática y su relación con el proceso Enseñanza-Aprendizaje (E-A) informan que tal proceso resulta exitoso cuando el profesor usa diversos modelos como estrategia didáctica para desarrollar el currículo (Peña y Morales, 2016) tanto en el ámbito de la educación básica como de la universitaria; tales modelos pueden generar actitudes y percepciones positivas en los estudiantes cuando se plasman los datos del contexto real en un modelo matemático (Özdemir \& Üzel, 2012). En el modelaje de fenómenos reales con presencia de incertidumbre o azar es conveniente usar estrategias que involucren la recogida de datos, el razonamiento probabilístico, el manejo de variables aleatorias y de distribuciones de probabilidad (Pantoja et al., 2015).

La modelación matemática en sí misma se constituye en una estrategia didáctica en tanto que posibilita crear, ajustar o usar modelos matemáticos por medio del estudio de problemas reales en un determinado contexto (Zaldívar et al., 2017); sin embargo, tanto en los estudiantes como docentes, investigadores y profesionales en ejercicio se observa una baja capacidad para llevar a la práctica la implementación de modelos a partir de datos, en particular, cuando se trata de datos asociados con variables aleatorias y especialmente en el modelaje de líneas de espera en sistemas reales, en los cuales, los usuarios han de hacer colas para acceder a un servicio; en este contexto, la pregunta de investigación que orientó este trabajo fue ¿Cuál estrategia didáctica se ha de implementar para ajustar modelos probabilísticos en el ámbito de los sistemas de colas o líneas de espera?

Para implementar tal estrategia se propone abordar las siguientes fases: i) revisión de aspectos conceptuales referidos al proceso general de modelación matemática y sobre la teoría de colas, ii) generación de un algoritmo para ajustar modelos probabilísticos, iii) aplicación de los modelos a un caso práctico.

\subsection{ASPECTOS CONCEPTUALES}

En general, la modelación matemática puede concebirse como un proceso que involucra un conjunto secuenciado de actividades destinado a construir un modelo matemático a partir de un problema o situación real (Blum, 1993); según Vasco (2003) este proceso puede incluir varios momentos, entre ellos, la creación del modelo, la puesta en funcionamiento y la revisión del 
mismo. De acuerdo con Trigueros (2006) se trata de un proceso cíclico que involucra tres momentos: la introducción al contexto real, la matematización del problema en estudio y las síntesis con retorno al contexto real; además, Ochoa y Vahos (2009) agregan que el ciclo puede iniciar con la determinación de un problema real, el cual ha de ser observado y en lo posible sometido a experimentación para comprenderlo mejor y recoger los datos que se produzcan, se prosigue a representar la realidad de forma simplificada mediante el ajuste de un modelo analítico $u$ otros y se generan conclusiones que se han de interpretar a la luz de dicho problema.

Por otra parte, un sistema puede ser modelado o simulado a través de un conjunto estructurado de elementos (Pantoja et al., 2015); para ello, una estrategia consiste en implementar los siguientes pasos: i) observar el sistema ii) plantear el problema, iii) recoger datos, iv) formular modelos matemáticos, v) estimar parámetros, y vi) evaluar los modelos propuestos (Naylor et al., 1977). En específico, los sistemas de colas incluyen elementos como los clientes (usuarios), un servicio por prestar y una capacidad operativa instalada; sus líneas de espera pueden modelarse mediante variables aleatorias y su distribución de probabilidad o con procesos de simulación (Jáuregui et al., 2017); en este tipo de sistema interesa modelar la tasa de servicio, los tiempos de espera y la eficiencia en la operación de tal sistema (Pérez et al., 2019).

En el mundo real, con frecuencia los individuos tienen que hacer cola o fila para obtener cierto servicio o producto, como pagar en el supermercado o comprar un tiquete para almorzar o entrar al cine (Vergara, 2019); una herramienta matemática usada para modelar líneas de espera es la teoría de colas, la cual proporciona variados modelos probabilísticos para analizar el comportamiento de diversos sistemas de colas (Piratelli et al., 2015); para ajustar dichos modelos puede recurrirse a técnicas estadísticas (Yifan, 2016) una vez se han colectado los datos referidos a los tiempos que los usuarios esperan en la cola o los tiempos que el sistema se tarda en servirlos (Taha, 2011); para optimizar la operación del sistema y minimizar los costos se han de diseñar y poner en marcha modelos de colas adecuados (Bohórquez y Fernández, 2017). En este contexto, los puntos de venta de los tiquetes para el almuerzo estudiantil subsidiado (kioscos universitarios) prestan un servicio al estudiantado que amerita ser analizado con modelos para líneas de espera.

De acuerdo con Gross \& Harris (2014) un sistema de colas involucra los siguientes elementos: i) los clientes por atender, ii) las llegadas o número de clientes en la cola de servicio; al número promedio de llegadas por unidad de tiempo se le denomina tasa media de llegadas $(\lambda)$; el tiempo esperado entre llegadas es $1 / \lambda$, iii) la tasa de servicio o capacidad instalada de operación, la cual incluye uno o varios servidores, iv) la tasa media de servicio o promedio de clientes que se atienden por unidad de tiempo $(\mu)$; el tiempo esperado de servicio es $1 / \mu, v$ ) el número de servidores, y vi) la línea de espera con un protocolo de servicio. Según Hillier \& Lieberman (2010) y García (2016) en el modelaje de tal sistema se busca un patrón de llegada y de servicio de los clientes de acuerdo con una disciplina de colas (Coelho, 2010) y con el número de canales de servicio. En general tales patrones se han de modelar con distribuciones probabilísticas adecuadas (Taha, 2011).

En los sistemas de colas es común usar la notación de Kendall (1953) representada por $M / G / S$, donde $M$ indica la distribución de probabilidad de las llegadas, $\mathrm{G}$ es la distribución de servicio y $S$ corresponde al número de canales de servicio. Para modelar el tiempo de servicio puede usarse una distribución exponencial o una de Erlang, para el modelaje de las llegadas de 
los clientes se utiliza una distribución de Poisson y para analizar los tiempos entre llegadas se recurre a una distribución exponencial; sin embargo, también pueden utilizarse otras como una distribución Weibull (Vargas et al., 2020). En particular, algunos sistemas presentan una sola cola y un único servidor, denotados por M/G/1 tal como ocurre en el kiosco donde los estudiantes de la UPTC adquieren sus tiquetes para almorzar; además, las colas pueden suponerse finitas o infinitas, de acuerdo con la capacidad operativa del sistema y de su estabilidad (Anderson et al., 2011).

Para analizar un sistema de colas con estructura $M / G / 1$, se pueden establecer las siguientes medidas de rendimiento (Hillier \& Lieberman, 2002): i) Utilización promedio del sistema, ii) promedio de clientes en el sistema, iii) tiempo de espera previsto en el sistema, iv) el tiempo promedio de espera en la cola, $v$ ) promedio de clientes en la cola, vi) probabilidad de tener $n$ clientes en el sistema con $n=0,1,2,3, \ldots$ vii) probabilidad de que el tiempo de espera en el sistema $(\Psi)$ exceda cierta cantidad de tiempo $t$. Estas medidas tienen sentido solamente cuando $\rho<1$; en otro caso, el servidor carece de la capacidad de atender todas las llegadas y el sistema se encuentra en estado inestable (Hillier \& Lieberman, 2002).

\subsection{ANTECEDENTES}

En educación matemática, la modelación se ha constituido en una estrategia didáctica poderosa para potenciar el aprendizaje y desde diversos organismos tanto nacionales como internacionales se sugerido su inclusión en el currículo escolar y universitario (Ochoa y Vahos, 2009); además, por sus distintas aplicaciones, la teoría de colas ha sido útil para modelar líneas de espera presentes en diferentes procesos administrativos, económicos, ingenieriles, industriales y comunicativos entre otros (Vargas et al., 2020); incluso se ha avanzado hacia el modelaje por medio de procesos de simulación (Burbano et al., 2014; Estrada et al., 2019). En seguida, se indican algunos trabajos elaborados en el ámbito internacional. En un contexto educativo, Trigueros (2006) realiza un estudio para usar la modelación en el movimiento del péndulo; Peña y Morales (2016) trabajan la modelación matemática como estrategia de E-A para determinar el área bajo curvas.

Por su parte, Molina (2017) usa la modelación matemática y la tecnología como estrategia didáctica para enseñar algunos temas de cálculo. Plaza (2016) aplica la modelación matemática en ciencias básicas para ingeniería. Estrada et al. (2019) elaboraron un modelo híbrido de teoría de colas apoyado en un algoritmo evolutivo para optimizar el servicio en un local de comidas rápidas. Piratelli et al. (2015) hicieron el modelaje de las líneas de espera en una industria alimentaria por medio de distribuciones de probabilidad. Jáuregui et al. (2017) modelaron la sección de urgencia de un hospital; Lin et al. (2013) con un modelo de colas por etapas analizaron el flujo entre dos áreas hospitalarias. Zaldívar et al. (2017) usaron la modelación matemática en la formación de profesores.

En el ámbito colombiano, Pantoja et al. (2015) usaron modelos de simulación como un recurso didáctico; Vargas et al. (2020) utilizaron una distribución de probabilidad para modelar la entrega de refrigerios en una universidad; Bohórquez y Fernández (2017) analizaron las colas en una entidad financiera; Bernal et al. (2009) modelan los tiempos de espera mediante procesos de simulación con una hoja de cálculo; Arias et al. (2010) usan la teoría de líneas de espera para modelar los procesos de una empresa; Arroyo et al. (2014) proponen ciertas distribuciones de probabilidad para modelar tiempos de espera; Vergara (2019) usa la teoría de colas para modelar el servicio de consulta en una biblioteca universitaria. Estos antecedentes 
justifican implementar una estrategia didáctica para modelar un problema real en la cola del kiosco estudiantil de la UPTC.

\section{METODOLOGÍA}

Este trabajo se desarrolló bajo un enfoque cuantitativo de investigación (Hernández y Mendoza, 2018) basado en un diseño de corte transversal, centrado en el modelaje de las líneas de espera y servicio en el kiosco estudiantil de la UPTC. La estrategia implementada involucra los pasos expuestos por Naylor et al. (1977) y Gross \& Harris (2014) para modelar un sistema de colas, así: i) observar el sistema y determinar el tamaño de la muestra, ii) recoger y organizar datos empíricos, iii) formular modelos matemáticos, mediante un análisis exploratorio de datos empíricos se propusieron los modelos probabilísticos de Poisson y exponencial para analizar tanto los tiempos de espera en la cola del kiosco como los tiempos de servicio en la ventanilla del mismo, iv) estimación de parámetros, para esto, se definieron dos variables aleatorias discretas así, X: número de estudiantes que llegaron a la cola del kiosco en intervalos de 10 minutos en el horario de 9:30 a 11:00 a.m. en días de labor académica elegidos de manera aleatoria; Y: número es estudiantes que salían del kiosco, en el mismo intervalo de tiempo, v) especificación de los modelos, y vi) evaluación de los modelos y obtención de medidas de rendimiento.

El sistema de colas correspondiente al kiosco se observó cuando estaba en plena operación con su dinámica propia; en este sentido, se procedió al cálculo del tamaño de la muestra (Burbano y Valdivieso, 2016) que resultó conformado por 104 intervalos de 10 minutos para las llegadas y servicios en el kiosco, la muestra provino de una población infinita de intervalos de tiempo, ya que el kiosco funciona de lunes a viernes durante el semestre académico; luego se recogió y organizó la información en una planilla elaborada para tal fin. Para el modelaje del sistema, se procedió así: i) se propuso ajustar un modelo de Poisson para los datos de la variable $X$, ii) se ajustó otro modelo de Poisson para los datos de la variable $Y$, con el uso de una distribución exponencial para modelar los tiempos de servicio (Clarke, 1957); iii) se determinaron los estimadores de los modelos por medio del método de máxima verosimilitud (Clarke, 1957; Burbano et al., 2019); iv) se hicieron las pruebas de bondad de ajuste, las hipótesis se contrastaron con un nivel de significancia del 5\% (Taha, 1991; Freund y Miller, 2000); v) se calcularon medidas de rendimiento (Taha, 2004; Hillier \& Lieberman; 2002; Gross \& Harris, 2014).

El análisis estadístico fue elaborado con el software libre $\mathrm{R}$, el cual posibilitó generar tablas que contienen estadísticas como promedio, desviación estándar (DS), máximo, mínimo, coeficiente de variación (CV) y el cálculo de algunas medidas de rendimiento a partir de los modelos ajustados. Los datos fueron recogidos en el mes de noviembre de 2019 con el consentimiento de Bienestar Universitario de la UPTC y usados solo con fines académicos e investigativos (Ley Habeas Data). El kiosco estudiantil objeto de estudio es una caseta provista de una ventanilla y con la infraestructura necesaria para atender a más de 1500 estudiantes en la jornada diurna en diversos horarios; los datos usados en el modelaje se recolectaron de 9:30 a.m. a 11:00 p.m.

\section{RESULTADOS}

En esta sección se indican los resultados referidos del modelaje realizado en el kiosco, éstos se han organizado en tres partes, en la primera se hace el modelaje de las llegadas a la cola del kiosco; en la segunda, se implementa los modelos asociados con el servicio de venta de los tiquetes de almuerzo en la ventanilla del kiosco, y en la tercera, se determinan algunas medidas de rendimiento del sistema de colas. 


\subsection{LLEGADAS A LA COLA DEL KIOSCO}

Acudiendo a una muestra piloto se calculó el tamaño de la muestra (Burbano y Valdivieso, 2016) a través de la expresión:

$n=\frac{Z^{2} \hat{S}^{2}}{e^{2}}$

El pilotaje se efectuó con una muestra conformada por 11 datos asociados con la variable $\mathrm{X}$; obteniéndose un promedio de $36 \mathrm{y}$ una DS corregida con valor de 3.93; para un nivel de confianza del $95 \%$ se obtuvo el valor $Z=$ 1.96; siguiendo el criterio de los investigadores, se decidió trabajar con un error absoluto de 2.1 $\%$ (0.021) del promedio muestral obtenido; por lo tanto se asumió un $e=0.021(36)=0.756$.

Así entonces,

$$
n=\frac{Z^{2} \hat{S}^{2}}{e^{2}}=\frac{(1.96)^{2}(3.93)^{2}}{(0.756)^{2}}=\frac{(3.8416)(15.45)}{0.5715}
$$

$$
n=\frac{59.3527}{0.5715}=103.55 \cong 104
$$

Tabla 1. Número de estudiantes que llegan a la cola del kiosco estudiantil en cada intervalo de 10 minutos

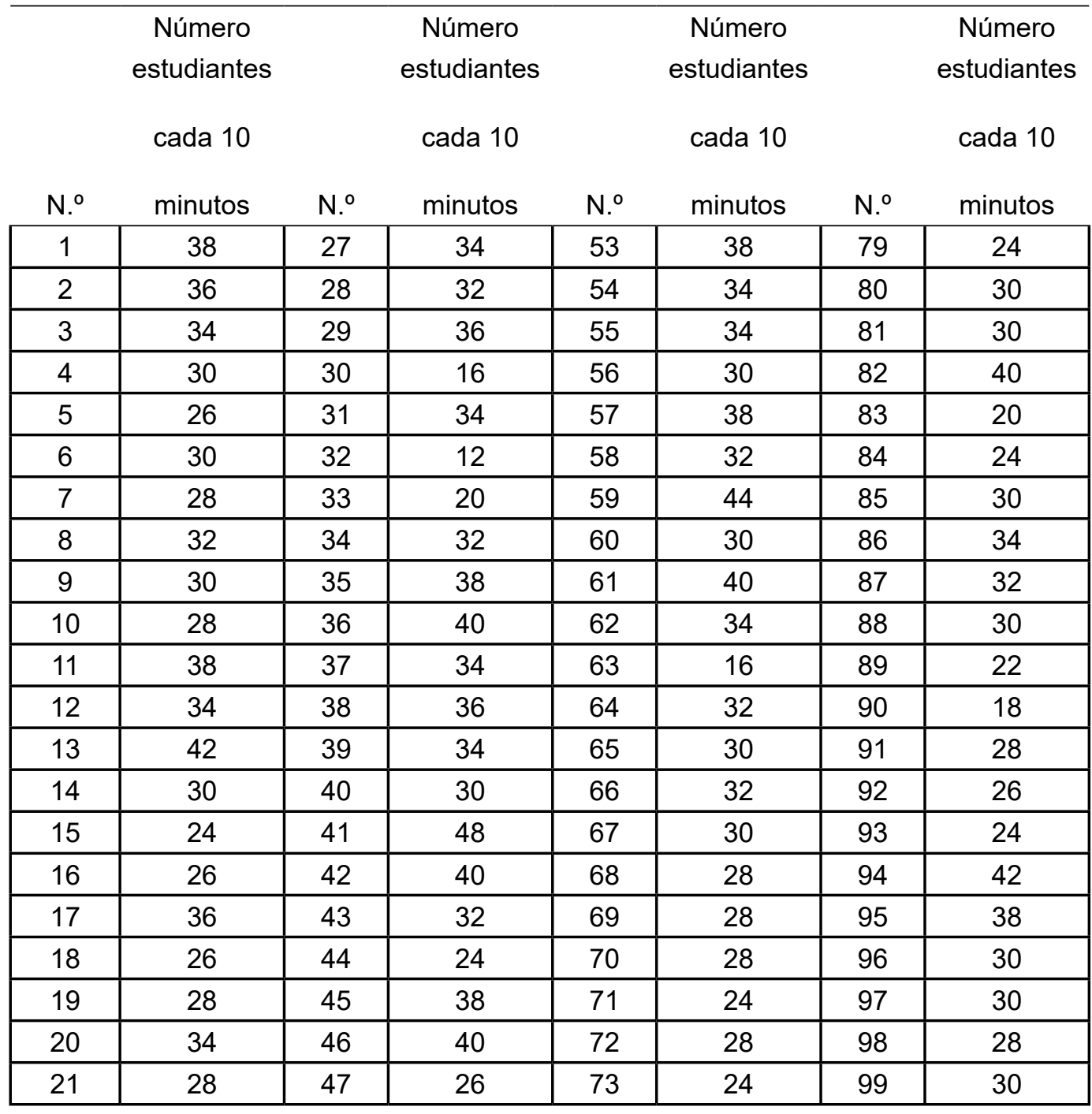




\begin{tabular}{|l|l|l|l|l|l|l|l|}
\hline 22 & 34 & 48 & 26 & 74 & 30 & 100 & 32 \\
\hline 23 & 32 & 49 & 32 & 75 & 24 & 101 & 32 \\
\hline 24 & 26 & 50 & 36 & 76 & 28 & 102 & 44 \\
\hline 25 & 20 & 51 & 26 & 77 & 30 & 103 & 34 \\
26 & 14 & 52 & 26 & 78 & 32 & 104 & 40 \\
\hline
\end{tabular}

Fuente: elaborada por los autores (2021)

Luego, el tamaño de la muestra resultante fue de 104 intervalos de tiempo. Para el modelaje de los datos, se partió del hecho de que el kiosco estudiantil estaba operando de forma estable con dinámica propia y poseía una capacidad instalada adecuada para atender a más de 15000 estudiantes por semana al acumular los atendidos en la jornada de la mañana y noche (Taha, 1991). Los datos recolectados para la variable $\mathrm{X}$ se indican en la Tabla 1.

A través de un análisis exploratorio se determinó que los datos de la variable aleatoria $X$ podrían modelarse con una distribución de Poisson; en este contexto, se procedió a estimar el parámetro de tal distribución siguiendo el procedimiento indicado por Burbano et al. (2019), se determinó que, el estimador de máxima verosimilitud para el respectivo parámetro en la distribución de Poisson es:

$$
\hat{\lambda}=\frac{1}{n} \sum_{i=1}^{n} x_{i}
$$

Con los datos de la Tabla 1 y los resultados de la Tabla 2, el valor del estimador fue:

$$
\hat{\lambda}=\frac{1}{104} \sum_{i=1}^{104} x_{i}=\frac{3196}{104}=30.73
$$

Tabla 2. Estadísticas para los datos en la cola del kiosco estudiantil

\begin{tabular}{l} 
n: tamaño de la muestra \\
\begin{tabular}{|l|r|}
\hline Promedio & 104 \\
\hline DS & 30.73 \\
\hline Mínimo & 6.53 \\
\hline Máximo & 12 \\
\hline
\end{tabular} \\
\hline
\end{tabular}

Fuente: elaborada por los autores (2021)

De acuerdo con la Tabla 2, el número de usuarios que llegaron a la cola del kiosco estudiantil varió desde 12 hasta 48 para los 104 intervalos de tiempo de 10 minutos. El promedio de estudiantes que llegaron a la cola del kiosco fue de 30.73 , esto indica que llegaron 31 usuarios cada 10 minutos, aproximadamente. La DS fue 6.53 , el CV $=6.53 / 30.73=0.2124=21.24$ $\%$, este porcentaje refleja que el número de estudiantes que llega a la cola cada 10 minutos, fue casi heterogéneo (Burbano y Valdivieso, 2016); es decir, presentaron una considerable variabilidad.

Se continuó con la aplicación de un algoritmo de la prueba K-S de Kolmogorov - Smirnov para establecer la bondad del ajuste de los datos a una distribución de Poisson, en la cual, el parámetro sea $\lambda=30.73$, los pasos fueron:

i) Proponer la función de probabilidad de Poisson para modelar las llegadas a la cola:

$$
f(x)= \begin{cases}\frac{\lambda^{x} e^{-\lambda}}{x !} & \text { Si } x=0,1,2,3, \ldots \\ 0 & \text { En otro caso }\end{cases}
$$


ii) Plantear la hipótesis nula y alternativa con un nivel de significancia del $5 \%$ así:

Ho: los datos se ajustan a la distribución de

Poisson de parámetro $\lambda=30.73$

$\mathrm{H}_{1}$ : los datos no se ajustan a la distribución de

Poisson de parámetro $\lambda=30.73$

iii) A través del paquete $R$, se determinó un $p$-valor $=0.728$, así entonces, de acuerdo con Burbano et al. (2019), se cumple que:

p-valor $=P\left(D>D_{o b s} /\right.$ Ho es cierta $)=0.728$

vi) Con base en la prueba K-S, se concluyó que los datos de la variable $X$ sí se ajustaban a una distribución de Poisson al $5 \%$ de significación ya que el $p$-valor obtenido de 0.728 resultó superior a 0.05 , luego se acepta $\mathrm{H}_{0}$ : los datos provienen de una distribución de Poisson con parámetro $\lambda=30.73$. Adicionalmente, una prueba chi-cuadrado ratificó la aceptación de la hipótesis nula con un $p$-valor de 0.0001 (chicuadrado=80.154).

En este contexto, el modelaje del fenómeno aleatorio de las llegadas a la cola del kiosco se puede hacer con un proceso de Poisson así (Gross \& Harris, 2014):

$$
P_{x}(t)=\frac{(\lambda t)^{x} e^{-\lambda t}}{x !} \quad \text { Con } x=0,1,2,3, \ldots
$$

Con $\lambda=30.73$, resulta:

$P_{x}(t)=\frac{(30.73 t)^{x} e^{-30.73 t}}{x !} \quad$ Con $x=0,1,2,3, \ldots$

Siguiendo a Gross \& Harris (2014), cuando el proceso de llegadas de los usuarios se ajusta a un proceso de Poisson de parámetro $\lambda t$, entonces la distribución de los tiempos entre llegadas corresponde a una distribución exponencial de parámetro $\lambda$, así:

$$
f(t, \lambda)=\left\{\begin{array}{lll}
\lambda e^{-\lambda t} & \text { si } & t>0 \\
0 & \text { si } & t \leq 0
\end{array}\right.
$$

Para el valor $\lambda=30.73$, se obtiene:

$$
f(t, 30.73)=\left\{\begin{array}{lll}
30.735 e^{-30.73 t} & \text { si } & t>0 \\
0 & \text { si } & t \leq 0
\end{array}\right.
$$

En este modelo, $\lambda=30.73$ hace referencia a la tasa de llegadas de los usuarios a la cola; luego, en promedio 31 estudiantes aproximadamente llegan a la cola del kiosco cada 10 minutos. En consecuencia, el promedio de estudiantes que llega por hora, se obtiene haciendo $t=6$; es decir, $\lambda t=30.73(6)=184.38$; así entonces, aproximadamente 134 estudiantes llegan al kiosco entre las 9:30 a.m. y las 11:00 a.m. para comprar su tiquete de almuerzo.

Siguiendo a Taha (2011) y Gross \& Harris (2014), el promedio del tiempo entre llegadas es $E(t)=$ $1 / \lambda=1 / 30.73=0.0325$; este resultado evidencia que el tiempo promedio entre llegadas es de 0.0325 de 10 minutos; es decir, 0.0325 (10) = 0.325 minutos aproximadamente, de forma análoga, 0.325 minutos $=0.325(60)$ segundos $=19.5$ segundos; por esto, el tiempo entre llegadas de los estudiantes al kiosco resultó ser relativamente corto.

\subsection{SALIDAS DEL KIOSCO ESTUDIANTIL}

De forma paralela al proceso de registro de las llegadas a la cola del kiosco, se recolectaron los datos de la variable $Y$, en intervalos de 10 minutos, referidos al número de usuarios atendidos en la ventanilla del kiosco, datos se indican en la Tabla 3. 
Tabla 3. Número de estudiantes atendidos en cada intervalo de 10 minutos

\begin{tabular}{|c|c|c|c|c|c|c|c|}
\hline N. ${ }^{\circ}$ & Atendidos & N. ${ }^{\circ}$ & Atendidos & N. ${ }^{\circ}$ & Atendidos & N. ${ }^{\circ}$ & Atendidos \\
\hline 1 & 40 & 27 & 42 & 53 & 39 & 79 & 30 \\
\hline 2 & 38 & 28 & 36 & 54 & 36 & 80 & 35 \\
\hline 3 & 37 & 29 & 42 & 55 & 43 & 81 & 30 \\
\hline 4 & 33 & 30 & 29 & 56 & 42 & 82 & 40 \\
\hline 5 & 34 & 31 & 37 & 57 & 39 & 83 & 37 \\
\hline 6 & 33 & 32 & 46 & 58 & 43 & 84 & 39 \\
\hline 7 & 37 & 33 & 38 & 59 & 48 & 85 & 31 \\
\hline 8 & 36 & 34 & 39 & 60 & 36 & 86 & 39 \\
\hline 9 & 35 & 35 & 37 & 61 & 40 & 87 & 35 \\
\hline 10 & 38 & 36 & 44 & 62 & 39 & 88 & 35 \\
\hline 11 & 37 & 37 & 43 & 63 & 31 & 89 & 33 \\
\hline 12 & 36 & 38 & 39 & 64 & 34 & 90 & 32 \\
\hline 13 & 40 & 39 & 35 & 65 & 36 & 91 & 39 \\
\hline 14 & 36 & 40 & 37 & 66 & 34 & 92 & 35 \\
\hline 15 & 32 & 41 & 38 & 67 & 42 & 93 & 35 \\
\hline 16 & 42 & 42 & 43 & 68 & 38 & 94 & 42 \\
\hline 17 & 42 & 43 & 41 & 69 & 34 & 95 & 39 \\
\hline 18 & 29 & 44 & 30 & 70 & 35 & 96 & 38 \\
\hline 19 & 33 & 45 & 33 & 71 & 30 & 97 & 37 \\
\hline 20 & 39 & 46 & 39 & 72 & 39 & 98 & 33 \\
\hline 21 & 38 & 47 & 38 & 73 & 34 & 99 & 32 \\
\hline 22 & 40 & 48 & 29 & 74 & 32 & 100 & 36 \\
\hline 23 & 37 & 49 & 37 & 75 & 34 & 101 & 37 \\
\hline 24 & 38 & 50 & 38 & 76 & 36 & 102 & 40 \\
\hline 25 & 28 & 51 & 38 & 77 & 36 & 103 & 35 \\
\hline 26 & 24 & 52 & 36 & 78 & 35 & 104 & 42 \\
\hline
\end{tabular}

Fuente: elaborada por los autores (2021)

Para los datos de la variable $\mathrm{Y}$, se calcularon algunas medidas estadísticas y con un análisis exploratorio se obtuvieron las primeras evidencias de que dichos datos también se podían modelar con una distribución de Poisson. Se estableció que el estimador de máxima verosimilitud era (Burbano et al., 2019):

$$
\hat{\mu}=\frac{1}{n} \sum_{i=1}^{n} x_{i}=\frac{1}{104} \sum_{i=1}^{104} x_{i}=\frac{3817}{104}=36.7
$$

Tabla 4. Estadísticas para los datos en la salida del kiosco estudiantil

\begin{tabular}{|c|c|}
\hline n: tamaño de la muestra & 104 \\
\hline Promedio & 36.7 \\
\hline DS & 4.093 \\
\hline Mínimo & 24 \\
\hline Máximo & 48 \\
\hline
\end{tabular}

Fuente: elaborada por los autores (2021) 
El número de estudiantes atendidos varió desde 24 hasta 48 para los 104 intervalos de 10 minutos (ver Tabla 4); el promedio fue de 36.7, este valor indica que aproximadamente 37 usuarios fueron atendidos cada 10 minutos. La DS fue de 4.093 aproximadamente. El CV $=4.093 / 36.7=0.1115$ $=11.15 \%$ indica que, los datos de la variable $\mathrm{Y}$, fueron casi homogéneos; es decir, se observó en éstos poca variación.

Nuevamente se usa la prueba K-S para determinar la bondad del ajuste de los datos a un modelo de Poisson con parámetro $\mu=36.7$ :

i) Se propuso el modelo de Poisson para los estudiantes atendidos, así:

$$
f(x)=\left\{\begin{array}{lc}
\frac{\mu^{x} e^{-\mu}}{x !} & \text { Si } x=0,1,2,3, \ldots \\
0 & \text { En otro caso }
\end{array}\right.
$$

ii) Se plantearon las hipótesis por probar con un nivel de significancia del $5 \%$, así:

Ho: los datos se ajustan a la distribución de Poisson de parámetro $\mu=36.7$

$\mathrm{H}_{1}$ : los datos no se ajustan a la distribución de Poisson de parámetro $\mu=36.7$

iii) A través del paquete $R$, se obtuvo un $p$-valor $=0.18$, luego según Burbano et al. (2019), se verifica que:

$$
p \text {-valor }=P\left(D>D_{\text {obs }} / \text { Ho es cierta }\right)=0.18
$$

vi) Se concluye que los datos si se ajustaban a un modelo de Poisson con una significancia del 0.05 puesto que el $p$-valor resultante fue de 0.18 y es superior a 0.05 ; en consecuencia se acepta que los datos se han de modelar con distribución de Poisson de parámetro $\mu=36.7$. Además, una prueba chi-cuadrado ratificó la aceptación de la hipótesis nula con un $p$-valor de 0.0002 (chi-cuadrado=58.692).
En consecuencia, el modelo probabilístico para los datos de la variable $\mathrm{Y}$, corresponde a un modelo de Poisson definido así:

$$
P_{y}(t)=\frac{(\mu t)^{y} e^{-\mu t}}{y !} \quad \text { Con } y=0,1,2,3, \ldots
$$

Para $\mu=36.7$, se obtiene:

$P_{y}(t)=\frac{(36.7 t)^{y} e^{-36.7 t}}{y !} \quad$ Con $y=0,1,2,3, \ldots$

Siguiendo a Gross \& Harris (2014), cuando el proceso de servicio es de Poisson con parámetro $\mu t$, entonces la distribución de probabilidad de los tiempos de servicio se asocian con una distribución exponencial de parámetro $\mu$, la cual se puede definir como:

$$
f(t, \mu)=\left\{\begin{array}{lll}
\mu e^{-\mu t} & \text { si } & t>0 \\
0 & \text { si } & t \leq 0
\end{array}\right.
$$

Para $\mu=36.7$, resulta:

$$
f(t, 36.7)=\left\{\begin{array}{lll}
36.7 e^{-36.7 t} & \text { si } & t>0 \\
0 & \text { si } & t \leq 0
\end{array}\right.
$$

Por lo tanto, se tiene que $\mu=36.7$ es la tasa de servicio en la ventanilla del kiosco en los intervalos de tiempo considerados. Por consiguiente, en promedio 37 usuarios fueron atendidos aproximadamente cada 10 minutos. Para determinar el promedio por cada hora se toma $t=6$; es decir, $\quad \mu t=36.7(6)=220.2$ ; de forma análoga, aproximadamente 220 
estudiantes abandonan la ventanilla por hora una vez fueron atendidos.

De acuerdo con Taha (2011) y Gross \& Harris (2014), el tiempo promedio entre salidas del servicio es $E(t)=1 / \mu=1 / 36.7=0.0272$; este valor evidencia que el promedio de tal tiempo es de 0.0272 de un lapso de 10 minutos; es decir, $0.0272(10)=0.272$ minutos; de manera análoga, 0.272 minutos $=0.272(60)$ segundos $=16.32$ segundos. Por consiguiente, el tiempo promedio entre salidas es de aproximadamente 16.32 segundos.

\subsection{MEDIDAS DE RENDIMIENTO EN EL SISTEMA DE COLAS DEL KIOSCO}

Inicialmente se ha observado que el sistema real de colas del kiosco se encontraba operando con una dinámica propia, presentaba una capacidad operativa estable para atender a los clientes (Taha, 1991). Los parámetros estimados de los modelos probabilísticos ajustados tanto para la cola de llegada al kiosco como para la salida del mismo evidenciaron que, efectivamente la tasa de servicio fue superior a la tasa de llegada a la cola, lo cual ha garantizado la estabilidad del sistema de colas modelado.

Siguiendo Hillier \& Lieberman (2002) se calcularon las medidas de rendimiento indicadas en la Tabla 5 , de allí se interpreta que, i) la utilización promedio del sistema de colas del kiosco fue del $83.73 \%$; este valor corresponde a la tasa promedio de tiempo que emplea el servidor para atender a los usuarios; además, resultó que $\rho<1$, lo cual implica que el servidor si posee la capacidad de atender a todos los usuarios de la cola, ii) el número esperado de usuarios en el kiosco fue de 5.1463, iii) el tiempo promedio transcurrido en el sistema, incluido el tiempo de servicio fue de 0.1675 , iv) el tiempo promedio de espera en la cola, sin contar el tiempo de servicio, fue de 0.1402 , v) el número esperado de clientes en la cola fue de 4.31, vi) la probabilidad de tener exactamente $n=3$ estudiantes en el sistema, fue de 0.0955 (9.55\%), vii) la probabilidad de tener exactamente un solo usuarios en el sistema, fue de 0.1362 (13.62\%), viii) la probabilidad de que el tiempo de espera en el sistema $(\Psi)$ exceda a $t=1$, lapsos de tiempo de 10 minutos fue de 0.00255 . 
Tabla 5. Medidas de rendimiento del kiosco estudiantil

\begin{tabular}{|c|c|c|c|}
\hline Medida & Valor & Medida & Valor \\
\hline $\begin{array}{l}\text { Promedio de llegadas por unidad } \\
\text { de tiempo }\end{array}$ & $\lambda=30.73$ & $\begin{array}{l}\text { Tiempo promedio } \\
\text { de espera en la } \\
\text { cola }\end{array}$ & $W_{q}=\rho W=0.1402$ \\
\hline Tasa media de servicio & $\mu=36.7$ & $\begin{array}{l}\text { Promedio de } \\
\text { clientes en la cola }\end{array}$ & $L_{q}=\lambda W_{q}=4.31$ \\
\hline Utilización promedio del sistema & $\rho=\frac{\lambda}{\mu}=0.8373$ & $\begin{array}{l}\text { Probabilidad de } \\
\text { tener } n \text { clientes en } \\
\text { el sistema }\end{array}$ & $\begin{array}{r}P_{n}=(1-\rho) \rho^{n} \\
P_{3}=0.0955\end{array}$ \\
\hline $\begin{array}{l}\text { Número promedio de clientes en el } \\
\text { sistema }\end{array}$ & $L=\frac{\rho}{1-\rho}=5.1463$ & $\begin{array}{l}\text { Probabilidad de } \\
\text { tener } n=1 \text { clientes } \\
\text { en el sistema }\end{array}$ & $P_{1}=0.1362$ \\
\hline $\begin{array}{l}\text { Tiempo de espera previsto en el } \\
\text { sistema }\end{array}$ & $W=\frac{1}{\mu-\lambda}=0.1675$ & $\begin{array}{l}\text { Probabilidad de } \\
\text { que el tiempo de } \\
\text { espera en el } \\
\text { sistema }\end{array}$ & $\begin{array}{l}P(\Psi>t)= \\
\operatorname{Exp}(-\mu(1-\rho) t) \\
\quad P(\Psi>t=1)= \\
0.00255\end{array}$ \\
\hline
\end{tabular}

Fuente: elaborada por los autores (2021)

\section{DISCUSIÓN}

En este estudio se ha logrado el objetivo de implementar una estrategia didáctica para elaborar un modelaje del sistema de colas del kiosco estudiantil de la UPTC mediante modelos probabilísticos; los pasos incluidos en la estrategia se ajustan a los indicados por Naylor et al. (1977) y Gross \& Harris (2014); sin embargo, estos no son los únicos y podrían estructurase estrategias alternativas. Los modelos utilizados en este trabajo, corresponden a las distribuciones de Poisson y exponencial tanto para la variable $\mathrm{X}$ como para $\mathrm{Y}$; no obstante, es posible recurrir a otros modelos como lo hace Piratelli et al. (2015) con distribuciones de Weibull para modelar otros sistemas de colas. En este sentido, el modelaje matemático es un proceso enriquecedor para quienes participan en su elaboración pero que requiere llevarse a cabo de manera adecuada y cuidadosa.

Con fundamento en los elementos conceptuales de la teoría de colas, se ha establecido que el problema real correspondía a un sistema de la forma $M / G / 1$, aspecto que está ligado a lo expuesto por Kendall (1953); los datos de la variable $X$ fueron modelados con un proceso de Poisson de parámetro $\lambda t$, el cual se ha interpretado como la tasa de llegada de los usuarios al Kiosco por unidad de tiempo; el tiempo entre llegadas fue modelado con una variable continua con distribución exponencial de parámetro $\lambda$, resultados concordantes con lo expuesto por Taha (2011), Gross \& Harris (2014) y Arroyo et al. (2014), cuyo valor fue de 30.73 y representa la tasa de llegadas al kiosco estudiantil. La estimación de los parámetros se realizó por medio del método de máxima 
verosimilitud; sin embargo, esta no es la única vía y puede acudirse a otros métodos como lo exponen Burbano et al. (2019) y Martínez et al. (2021).

Por otra parte, los datos de la variable $Y$, referidos a los usuarios atendidos en la ventanilla del kiosco muestran que, también podían modelarse con un proceso de Poisson de parámetro $\mu t$, el cual ha representado la tasa de servicio en el kiosco por unidad de tiempo $t$; además, el tiempo de servicio fue modelado con una variable continua cuya distribución fue una exponencial de parámetro $\mu$; este modelado guarda relación con lo indicado por Taha (2011) y Gross et al. (2009); el valor obtenido fue de 36.7 que representa la tasa de servicio en el kiosco. Por consiguiente, se evidenció que, el kiosco estudiantil correspondía a un sistema estable que operaba con dinámica propia, con una tasa promedio de servicio de $36.7 t$, donde $t$ correspondía a periodos de 10 minutos. Solamente, cuando el modelo ha sido implementado se puede usar para valorar la eficiencia operativa del sistema en estudio.

La aplicación del modelo facilita aún más la comprensión del problema real, por ejemplo, a partir de la forma analítica del modelo, se pudo determinar que en promedio, 220 estudiantes aproximadamente son atendidos por hora en el kiosco estudiantil en el horario de 9:30 a.m. a 11:00 a.m. los días de labor académica en la UPTC, este hecho ratifica lo expuesto por Trigueros (2006) de agotar las etapas del ciclo de modelaje. El retorno a observar nuevamente el kiosco permitió identificar que había días con mayor concurrencia en el kiosco y el flujo de usuarios no era uniforme; sin embargo, si asume una relativa uniformidad, al operar el kiosco por una horas y media cada día, con una tasa de rendimiento del $83.73 \%$ estaría en capacidad de atender a 330 estudiantes en ese horario, lo cual significa un alto nivel de eficiencia, ya que a la semana atendería a más de 1650 estudiantes en esta jornada y posiblemente más de unos 3300 en las dos jornadas.

Con la estimación de los parámetros del modelo, se pudo calcular algunas medidas de rendimiento, las cuales indicaron que, el kiosco estudiantil era un sistema estable ya que la tasa de servicio resultó ser mayor que la tasa de llegada a la cola, determinándose que la utilización promedio del kiosco estuvo cerca del $83.73 \%$ y la tasa de tiempo ocioso fue solo del $16.27 \%$. El tiempo promedio transcurrido en el sistema, incluido el tiempo de servicio fue relativamente pequeño con un valor de 0.1675 . Estos hallazgos permiten caracterizar a el kiosco estudiantil como un sistema con un nivel alto de eficiencia, con medidas de rendimiento un poco menores que las obtenidas Estrada et al. (2019) y Piratelli et al. (2015) en el modelaje de otros sistemas de colas. Finalmente, el modelaje realizado aporta elementos de tipo didáctico para modelar otros problemas reales semejantes.

\section{CONCLUSIONES}

Una estrategia didáctica para modelar problemas reales asociados con las líneas de espera involucra varios elementos conceptuales y un conjunto cíclico de pasos que tanto los estudiantes y docentes como los profesionales en ejercicio e investigadores han de poner en juego al momento de construir modelos a partir de datos; en este contexto, se concluye que el modelamiento matemático es una herramienta potente para representar un sistema real, pero requiere poner en marcha una estrategia planificada que va de los datos hacia el modelo y de este nuevamente a la realidad; este proceso no es único y guarda relación con los pasos del método científico experimental, entre ellos, la observación, la formulación y prueba de hipótesis, la elaboración de inferencias y la obtención de conclusiones. 
Los pasos asumidos en la estrategia evidenciaron que, en el modelaje del sistema de colas del kiosco estudiantil de la UPTC, las distribuciones de Poisson y exponencial resultaron adecuadas; las llegadas a la cola del kiosco se puede modelar mediante un proceso de Poisson de parámetro $\lambda t$ y la distribución de los tiempos entre llegadas con una distribución exponencial de parámetro $\lambda$; además, el modelaje de la variable aleatoria referida al número de estudiantes que fueron atendidos en la ventanilla del kiosco, se ajusta a un proceso de Poisson de parámetro $\mu t$, que ha representado la tasa de atención en la ventanilla por unidad de tiempo $t$. Los modelos utilizados permitieron concluir que, el sistema presentaba una forma $M / G / 1$, se encontraba operando de manera estable con un alto porcentaje (83.73\%) de utilización por parte de los usuarios; sin embargo, aún era posible mejorarlo.

\section{REFERENCIAS BIBLIOGRÁFICAS}

Anderson, D., Sweeney, D., Williams, T., Camm, J., \& Martin, K. (2011). Métodos cuantitativos para los negocios (Onceava ed.). México: CENGAGE LEARNING.

Arias, M., Portilla, L. \& Fernández, S. (2010). La distribución de costos indirectos de fabricación, factor clave al costear productos. Scientia Et Technica, 16(45).

Arroyo, I., Bravo, L. C., Llinás, H., \& Muñoz, F. L. (2014). Distribuciones Poisson y Gamma: una discreta y continua relación. Prospectiva, 12(1), 99-107.

Bernal, J. J, Sánchez, J. F., Martínez, M. S. (2009). La hoja de cálculo como apoyo a la simulación de los fenómenos de espera con prioridades. Una aplicación a la sanidad. Cartagena: Asociación Española de Profesores Universitarios de Matemáticas aplicadas a la Economía y la Empresa (ASEPUMA).
Blum,W. (1993). Mathematical modelling in mathematics education and instruction. In Breiteig, T., Huntley, I. and Kaiser-Messmer, G., (Eds.), Teaching and learning mathematics in context, Chichester, UK: Ellis Horwood, pp. 3-14.

Bohórquez, G. E., \& Fernández, J. S. (2017). Evaluación de la satisfacción del cliente en el sector financiero del municipio de PamplonaColombia. FACE: Revista de la Facultad de Ciencias Económicas y Empresariales, 17(2), 196-205.

Burbano, V.M., Valdivieso, M. A. \& Salcedo, L. A. (2014). Simulación con modelos aleatorios. Conocimiento estadístico probabilístico y simulación. Tunja: Editorial UPTC.

Burbano, V., y Valdivieso, M. (2016). Inferencia Estadística Básica, apoyo al estudio independiente. Tunja, Colombia: Editorial UPTC.

Burbano, V., Valdivieso, M., y Burbano, A. (2019). Confiabilidad: un enfoque paramétrico en la práctica investigativa. Tunja, Colombia: Editorial UPTC.

Coelho, A. S. (2010). Aplicación de la teoría de colas y de la simulación al embarque de mineral de hierro y manganeso en la terminal marítima de Ponta de Madeira. Revista Gestão Industrial, 6(3), 63-78.

Clarke, A. B. (1957). "Maximum likelihood estimates in a simple queue". The Annals of Mathematical Statistics, 28, 1036-1040.

Estrada, R. C., Pin, M. P., Solórzano, A. T., y Cevallos, L. (2019). Aplicación de un modelo híbrido de teoría de colas y algoritmo evolutivo para medir la optimización en el servicio de atención al cliente en un local de comidas rápidas. Ecuadorian Science Journal, 3(1), 1522. 
Freund, J., y Miller, I. (2000). Estadística Matemática con aplicaciones. México: Prentice Hall.

García, J. P. (2016). Aplicando Teoría de colas en Dirección de operaciones. Valencia, España: Grupo Rogle.

Gross, D. \& Harris, C. M. (2014). Fundamentals of queueing theory. New York: John Wiley \& Sons.

Hernández, R., y Mendoza, C. (2018). Metodología de la investigación. Las rutas cuantitativa, cualitativa y mixta. Ciudad de México, México: Editorial McGraw Hill

Hillier, F. \& Lieberman, G. (2002). Métodos cuantitativos para administración. México: McGraw-Hill.

Hillier, F., \& Lieberman, G. (2010). Introducción a la investigación de operaciones (Novena ed.). México: Mc Graw Hill.

Jáuregui, G. R. R., Pérez, A. K. G., González, S. H., \& Ripalda, M. D. H. (2017). Análisis del servicio de urgencias aplicando teoría de líneas de espera. Contaduría y Administración, 62(3), 719-732.

Jiménez, F. A. G. (2011). Aplicación de teoría de colas en una entidad financiera: herramienta para el mejoramiento de los procesos de atención al cliente. Revista Universidad EAFIT, 44(150), 51-63.

Kendall, D. G. (1953). "Stochastic processes occurring in the theory of queues and their analysis by the method of imbedded Markov chains". Annals of Mathematical Statistics, 24, 338-354.

Lin, D., Patrick, J. \& Labeau, F. (2013). Estimating the waiting time of multi-priority emergency patients with downstream blocking. Health Care Management Science, 17(1), 1-12. http:// dx.doi.org/10.1007/s10729-013-9241-3
Martínez, M. A., Mondragón, E. I., y Vergel, M. (2021). Un enfoque didáctico para el estudio del algoritmo genético en la estimación de parámetros de modelos matemáticos. Revista Boletín Redipe, Vol. 10, №. 3, 2021, págs. 301316.

Molina, J. A. (2017). Experiencia de modelación matemática como estrategia didáctica para la enseñanza de tópicos de cálculo. Uniciencia, 31(2), 19-36.

Naylor, Th., Balintfy, J., y Chu, K. (1977). Técnicas de simulación en computadoras. Ed. Limusa, México.

Ochoa, J. A. V., y Vahos, H. M. R. (2009). Modelación en educación matemática: una mirada desde los lineamientos y estándares curriculares colombianos. Revista virtual Universidad católica del norte, 27(1), 1-21.

Özdemir, E. \& Üzel, D. (2012). Student opinions on teaching based on mathematical modelling. Procedia - Soc. Behav. Sci., 55, 1207-1214.

Pantoja, V. M. Á. B., Sosa, J. E. P., \& Miranda, M. A. V. (2015). Formas de usar la simulación como un recurso didáctico. Revista Virtual Universidad Católica del Norte, (45), 17-37.

Peña, L. M., y Morales, J. F. (2016). La modelación matemática como estrategia de enseñanza-aprendizaje: El caso del área bajo la curva. Revista Educación en Ingeniería, 11(21), 64-71.

Piratelli, C. L., Achcar, J. A., \& Sandrim, R. R. (2015). Análise dos tempos entre chegadas de lotes em uma indústria alimentícia. Ingeniare. Revista chilena de ingeniería, 23(4), 569-578.

Plaza, L. F. (2016). Modelación matemática en ingeniería. IE Revista de investigación educativa de la REDIECH, 7(13), 47-57.

Taha, H. (1991). Investigación de operaciones. México: Alfaomega 
Taha, H. (1998). Investigación de operaciones, una introducción. México: Pearson Educación.

Taha, H. (2004). Investigación de operaciones. México: Pearson Educación.

Taha, H. (2011). Investigación de operaciones. (9. ${ }^{a}$ ed.). México: Addison-Wesley.

Trigueros, M. (2006). Ideas acerca del movimiento del péndulo: un estudio desde una perspectiva de modelación. Revista Mexicana de Investigación Educativa, 11(31), 1207-1240.

Vargas, J. J., Causado, E., y Mercado, H. (2020). Estimadores Bayesianos de distribuciones Weibull aplicados a un modelo de líneas de espera G/G/s. Revista de Métodos Cuantitativos para la Economía y la Empresa, 30, 142-162.

Vasco, C. E. (2003). El pensamiento variacional y la modelación matemática. In Anais eletrônicos do CIAEM-Conferência Interamericana de Educação Matemática, Blumenau (Vol. 9, pp. 2009-2010).

Vergara, A. M. (2019). Aplicación de la Teoría de líneas de Espera en el servicio de Biblioteca de una Organización Educativa en CartagenaColombia. Saber, ciencia y libertad, 14(1), 171178.

Yifan, W. U. (2016). Analysis of Prediction of Traffic using Probability Statistical Theory. International Journal of Simulation--Systems, Science \& Technology, 17(42).

Zaldívar, J. D., Quiroz, S. A., y Medina, G. (2017). La modelación matemática en los procesos de formación inicial y continua de docentes. IE Revista de investigación educativa de la REDIECH, 8(15), 87-110. 